\title{
BMJ Open Prevalence of type 2 diabetes mellitus in women of childbearing age in Africa during 2000-2016: protocol of a systematic review and meta-analysis
}

\author{
Tawanda Chivese, ${ }^{1,2}$ Werfalli Mahmoud, ${ }^{1}$ Itai Magodoro, ${ }^{3}$ Andre P Kengne, ${ }^{4}$ \\ Shane A Norris, ${ }^{2}$ Naomi S Levitt ${ }^{5}$
}

To cite: Chivese $T$ Mahmoud W, Magodoro I, et al. Prevalence of type 2 diabetes mellitus in women of childbearing age in Africa during 2000-2016: protocol of a systematic review and meta-analysis. BMJ Open 2016;6:e012255.

doi:10.1136/bmjopen-2016012255

- Prepublication history and additional material is available. To view please visit the journal (http://dx.doi.org/ 10.1136/bmjopen-2016012255).

Received 12 April 2016 Revised 6 September 2016 Accepted 8 November 2016

CrossMark

For numbered affiliations see end of article.

Correspondence to

Tawanda Chivese;

tchivese@gmail.com

\begin{abstract}
Introduction: African women of childbearing age are increasingly being exposed to risk factors for type 2 diabetes mellitus (T2DM), most particularly obesity. A differentiating feature of diabetes in women of childbearing age is that the disease may affect the mother and the developing fetus. Apart from mapping the extent of the problem, understanding the prevalence of T2DM in African women of childbearing age can help to galvanise targeted interventions for reducing the burden of T2DM. This is a protocol for a systematic review aiming to assess the prevalence of and risk factors for T2DM in women of childbearing age (15-49 years) in Africa.
\end{abstract}

Methods and analyses: We will carry out a comprehensive literature search among a number of databases, using appropriate adaptations of the African search filter to identify diabetes prevalence studies, published from 2000 to 2016, among African women of childbearing age (15-49 years) according to the WHO definition. Full copies of articles identified through searches and considered to meet the inclusion criteria will be obtained for data extraction and synthesis. The analysis of the primary outcome (prevalent diabetes) will include two steps: (1) identification of data sources and documenting estimates and (2) application of the random-effects meta-analysis model to aggregate prevalence estimates and account for between-study variability in calculating the overall pooled estimates and $95 \% \mathrm{Cl}$ for diabetes prevalence. We will assess heterogeneity and publication bias using established methods. This systematic review will be reported according to the Preferred Reporting Items for Systematic reviews and Meta-Analyses Protocol (PRISMA-P) 2015.

Ethics and dissemination: Ethical approval is not required for this study, given that this is a protocol for a systematic review, which utilises published data. The findings of this study will be widely disseminated through peer reviewed publications and conference presentations.

Trial registration number: CRD42015027635.

\section{Strengths and limitations of this study}

- This research will provide a comprehensive overview of all the data on the prevalence of type 2 diabetes in women of childbearing age in Africa, using repeatable transparent robust systematic review methods

- Age-specific prevalence data may not be available for some studies.

\section{INTRODUCTION}

The number of people with diabetes has increased considerably from 108 million people in 1980 to 422 million in 2014 and is expected to reach 700 million by 2025 . Globally, the diabetes prevalence among women increased from $5.0 \%$ in 1980 to $7.9 \%{ }^{1}$ Notably, the growing global burden of diabetes is occurring predominantly in lowincome or middle-income countries where health systems, ravaged by infectious disease, are ill-equipped to deal with the new costly burden (estimated worldwide annual cost of US $\$ 825^{1}$ ). In Africa, there has been a rapid increase in the prevalence of diabetes, and consistent with other regions, $90 \%$ of cases are type 2 diabetes mellitus (T2DM).$^{2-4}$ For example, in Tanzania and Cameroon, repeated local surveys using similar methods revealed that T2DM increased by 6 -fold and 10 -fold in a decade, respectively. ${ }^{3}{ }^{5}$ This increase in prevalence has led to estimates by the International Diabetes Federation (IDF) that the number of people in the region with T2DM is expected to more than double by 2035 relative to $2013 .{ }^{6}$ Considerations for the huge human and economic burden that results from treating T2DM and its complications have led to calls by the IDF for the 
establishment of national diabetes programmes to better deliver prevention and control solutions. ${ }^{6}$ At the heart of these efforts is a drive to identify high-risk populations, context specific-risk factors and implementing effective interventions to prevent or delay the onset of T2DM.

Women of childbearing age, defined by the WHO as women aged between 15 and 49 years, ${ }^{7}$ are affected by T2DM in a unique way. If a woman has T2DM and becomes pregnant, her unborn child is at high risk of developing T2DM in adulthood, ${ }^{8}$ thereby accelerating the intergenerational risk of T2DM. Interventions to prevent and control T2DM in this group is further warranted given the important contribution women make to the social and economic development of nations, the health and well-being of their children and families. Furthermore, women are valuable conduits for introducing healthy lifestyles in their families and communities.

The modifiable risk factors for T2DM are on the rise in all populations. In particular, overweight and obesity, the main drivers of the T2DM epidemic, are increasing worldwide and especially so in women. ${ }^{9}$ In Africa, the increase in overweight and obesity is attributed to the nutrition transition and modernisation characterised by adoption of energy dense 'Western' diets and decreased physical activity, ${ }^{10}$ although the evidence linking these factors to obesity in Africa is sparse. Other context specific factors such as cultural practices where being overweight is associated with higher economic status ${ }^{11} 12$ may also be contributing to overweight and obesity in this group. Obesity is thought to result in T2DM through excess visceral fat deposition that often results in ectopic fat deposition in the liver and other abdominal organs, leading to insulin resistance. ${ }^{13}$ Epidemiological studies have demonstrated a strong association with up to $90 \%$ of T2DM being attributable to overweight and obesity. ${ }^{14}$ Worldwide, the proportion of women who are obese is marginally higher than that of men $(40 \%$ vs $38 \%){ }^{14}$ However, in low-income to middle-income countries, Africa included, more women are obese compared to men. ${ }^{9}$

Multiparity may increase a woman's risk of obesity ${ }^{15}$ and resultant T2DM, although the evidence is inconsistent. ${ }^{16}$ A higher gestational weight has also been, in turn, associated with increased risk of weight gain, and a consequent risk of overweight and obesity after pregnancy. ${ }^{17}$ In Africa, the data on weight gain after pregnancy appear to be even scarcer, although women tend to have more children, compared to those in the developed world and therefore go through multiple pregnancy cycles, ${ }^{18} 19$ with the associated incremental weight gain after each cycle.

Africa has the greatest global burden of HIV, with women of childbearing age the group most affected. ${ }^{20}$ As a consequence of the successful rollout of antiretroviral therapy (ART) in many African countries, a large number of women have access to ART and life expectancy has increased. ART, including the drugs in widespread use in the region, however, has been linked with T2DM risk, ${ }^{21}$ which may impact the prevalence of T2DM in women of childbearing age.

The existing studies in Africa have not been previously collated, although there are perceptions that the prevalence of T2DM in women of childbearing age may be on the increase in the continent. This research will provide information on patterns and the distribution of T2DM to policymakers and possibly identify priority areas for intervention. We hope that the identification of risk factors specific to the African women of childbearing age will improve the development of effective interventions to delay or prevent T2DM in the continent.

\section{Aim}

The aim of this systematic review is to assess the prevalence of and the risk factors for the development of T2DM in African women aged between 15 and 49 years as reported in studies during the period 2000 to 2016 . The lower cut-off of the year 2000 will be used as studies conducted earlier may have used different criteria for the diagnosis of T2DM.

\section{Objectives}

To achieve the above aim, the research objectives will be:

1. To estimate the prevalence of T2DM in women of childbearing age in Africa, as reported in studies during the years 2000 to 2016 .

2. To determine risk factors for T2DM in women of childbearing age in Africa, as reported in research studies conducted during the years 2000 to 2016.

\section{Research question}

This systematic review will answer the following question: what is the prevalence of and risk factors for T2DM in women of childbearing age in Africa as reported in studies published during the period 2000 to 2016 ?

\section{Study design}

This protocol is registered online on PROSPERO, the International prospective register of systematic reviews (http:www.crd.york.ac.uk/PROSPERO, registration no. CRD42015027635). The Preferred Reporting Items for Systematic reviews and Meta-Analyses (PRISMA) ${ }^{22}$ guidelines for reporting systematic reviews will be followed.

\section{Criteria for considering studies for review Types of studies}

The systematic review will include cross-sectional studies and any other observational studies that assessed the prevalence of and risk factors for T2DM.

\section{Studies inclusion criteria}

1. All published and unpublished cross-sectional and community-based studies during the period 2000 to 2016 are reporting the prevalence of, risk factors for, T2DM in women aged 15-49 years, where the sample of women in this age range was at least 100 . 
2. Diagnosis of T2DM should have been according to the WHO 1999 guidelines $^{23}$ or an equivalent.

3. No language restriction will be applied.

\section{Studies exclusion criteria}

Studies will be excluded if:

1. Used criteria not comparable to the WHO 1999 guidelines in diagnosing T2DM.

2. They are duplicate publications. In the case of duplicate publications, only one article that contains the most information will be included in the review.

3. They are narrative review, letters to the editor, opinions or other publications that do not have primary data.

4. Studies in migrant Africans.

\section{Types of outcomes}

The prevalence of the following will be assessed from studies included in the systematic review.

\section{Primary outcomes}

T2DM as diagnosed according to the WHO 1999 guidelines: fasting blood glucose of at least $7.0 \mathrm{mmol} / \mathrm{L}$ or 2-hour oral glucose tolerance test (OGTT) blood glucose of $11.1 \mathrm{mmol} / \mathrm{L}^{23}$

\section{Secondary outcomes}

Impaired glucose regulation (IGR), which will be defined as either impaired glucose tolerance (fasting blood glucose $<7.0 \mathrm{mmol} / \mathrm{L}$ and 2-hour OGTT blood glucose of at least $7.8 \mathrm{mmol} / \mathrm{L}$ but $<11.1 \mathrm{mmol} / \mathrm{L}$ ) or impaired fasting glucose (fasting blood glucose $>6.1 \mathrm{mmol} / \mathrm{L}$ but $<7.0 \mathrm{mmol} / \mathrm{L}){ }^{23}$

\section{Search strategy for identification of relevant studies \\ Data sources}

The following sources will be searched for studies conducted during the period January 2000 to March 2016:

\section{Electronic databases}

1. Electronic databases including PubMed-MEDLINE, the Cochrane Central, Global Health, Scopus, CINAHAL, ISI web of science and POPLINE and AfricaWide

2. Grey literature databases such as OpenSigle

\section{Hand searching}

1. All references of retrieved articles will be scanned for further studies.

2. Prominent authors of articles will be contacted for information on other studies they may know.

\section{Search methods for identification of studies}

A comprehensive and sensitive search strategy using an African search filter will be utilised to identify research articles from the year 2000 to 2016 (see online supplementary appendix 1). An expert librarian will be consulted during the design of the search strategy.
Individual African country names and regional grouping names, such as sub-Saharan Africa and North Africa, will also be used to identify studies that may have been indexed under regional names. For countries with non-English as well as English names, both names will be used during searching while countries that have changed names during the period 2000-2016 will have all the names included in the search. Medical Subject Headings (MeSH) terms will be used when searching for studies in MEDLINE and PubMed. Endnote 7 will be used to manage retrieval of articles and screening for duplicates.

\section{Procedure for selection of studies}

Articles retrieved from the search will be exported to Endnote X7 where duplicates will be identified. Two investigators will screen titles, abstracts and if necessary full articles for inclusion. The full articles will then be screened for eligibility independently by the two investigators. If the investigators do not agree, a third investigator will be consulted. Trained interpreters will translate articles in languages other than English and French into English.

\section{Assessment of the quality of and risk of bias in included} studies

Two investigators will independently assess the included articles for the risk of bias and quality. They will resolve any differences by discussion and a third investigator will be consulted if they fail to reach consensus. Included studies will be assessed for quality (internal and external validity as well as risk of bias) using the validated quality appraisal tool developed by Hoy et al. ${ }^{24}$

\section{Data extraction and management}

After the studies have been assessed for risk of bias, two authors will independently extract data from the selected articles into a predefined data extraction form in Microsoft Office Excel 2016, which will first be piloted using five studies. The two investigators will compare their findings and discuss to resolve any differences.

Data to be extracted from the articles will include author names, date of publication, country where study was conducted, number of participants included and proportion of participants who were women of childbearing age, main findings, study design, language, sampling method, response rate, risk factors for T2DM and unadjusted T2DM prevalence estimates which will be extracted as the number of cases (denominator) out of the number in each age group (numerator).

We will use a predefined data extraction form in Microsoft Office Excel 2016, which will first be piloted using five studies.

We will contact authors to get information on agespecific prevalence should it not be reported. 


\section{Data synthesis and analysis}

For data that we are unable to conduct a meta-analysis, we will provide a narrative description. These data will include study characteristics such as year of publication, sample size and country where study and attributes associated with T2DM in women of childbearing age.

We will recalculate unadjusted estimates of the prevalence of T2DM and IGR among women within the age groups of (15-49) years (number of cases/sample size) together with SEs based on the information on crude numerators and denominators provided in the individual studies. We will pool the T2DM prevalence using the statistical software R V.3.2.3, ( $\mathrm{R}$ Core Team. R: A language and environment for statistical computing. R Foundation for Statistical Computing, Vienna, Austria. 2015; http:// www.R-project.org/ (accessed 30 Mar 2016)) and applying the appropriate variance stabilising transformations.

Should included studies not have age-specific prevalence of T2DM we will write to the authors and request the data.

We will assess heterogeneity between studies using Cochran's Q statistic. ${ }^{25}$ The Q-statistic tests for heterogeneity based on the null hypothesis that all studies share a common effect size. We will do the hypothesis testing based on a 0.10 level of significance, that is a $p$ value of $<0.10$, implying that studies do not share a common effect size. To estimate the percentage of total variation across studies due to true between-study differences rather than chance, we will use the $\mathrm{I}^{2}$ statistic $(<25 \%$ as low, between $25 \%$ and $50 \%$ as moderate and $>75 \%$ high heterogeneity). ${ }^{25}{ }^{26}$ Sources of heterogeneity will be explored through subgroup analysis using study-level characteristics such as geographical regions, rural/urban settings, age groups, study period, year of publication and sample sizes. This will be complemented where relevant, by meta-regression to further explain the heterogeneity, if any.

\section{Assessment of publication bias}

We will assess the presence of publication bias examining the funnel plots, supplemented with a formal statistical testing using the Egger test, ${ }^{27}$ and the Begg's test ${ }^{28}$ for publication bias. To test the robustness of our findings to publication bias, we will apply the Duval and Tweedie's trim and fill methods. ${ }^{29}$

\section{Author affiliations}

${ }^{1}$ Chronic Disease Initiative for Africa, Department of Medicine, University of Cape Town, South Africa

${ }^{2}$ Faculty of Health Sciences, South Africa Medical Research Council

Developmental Pathways for Health Research Unit, Department of Paediatrics, University of the Witwatersrand, Johannesburg, South Africa

${ }^{3}$ Harvard Medical School, Boston, USA

${ }^{4}$ Non-communicable Disease Research Unit, South African Medical Research

Council, Cape Town, South Africa

${ }^{5}$ Division of Endocrinology, Department of Medicine, University of Cape Town, Cape Town, South Africa

\section{Twitter Follow Tawanda Chivese @TChivese}

Contributors TC contributed to conception of the study, and drafted and revised the manuscript. He is the guarantor of the review. IM and WM revised the manuscript. APK revised the manuscript and approved the final submission. SAN and NSL contributed to conception of the study, and revised and approved the final submission.

Funding This research received no specific grant from any funding agency in the public, commercial or not-for-profit sectors.

Competing interests None declared.

Ethics approval This review will use secondary data from published and unpublished studies and will not require ethical clearance.

Provenance and peer review Not commissioned; externally peer reviewed.

Open Access This is an Open Access article distributed in accordance with the Creative Commons Attribution Non Commercial (CC BY-NC 4.0) license, which permits others to distribute, remix, adapt, build upon this work noncommercially, and license their derivative works on different terms, provided the original work is properly cited and the use is non-commercial. See: http:// creativecommons.org/licenses/by-nc/4.0/

\section{REFERENCES}

1. NCD Risk Factor Collaboration (NCD-RisC). Worldwide trends in diabetes since 1980: a pooled analysis of 751 population-based studies with 4.4 million participants. Lancet 2016;387:1513-30.

2. Peer N, Steyn K, Lombard C, et al. Rising diabetes prevalence among urban-dwelling black South Africans. PLOS ONE 2011;7: e43336.

3. Mbanya JCN, Motala AA, Sobngwi E, et al. Diabetes in sub-saharan Africa. Lancet 2010;375:2254-66.

4. Peer N, Kengne AP, Motala AA, et al. Diabetes in the Africa Region: an update. Diabetes Res Clin Pract 2014;103:197-205.

5. Aspray TJ, Mugusi F, Rashid S, et al. Rural and urban differences in diabetes prevalence in Tanzania: the role of obesity, physical inactivity and urban living. Trans $R$ Soc Trop Med Hyg 2000:94:637-44.

6. International Diabetes Federation. IDF Diabetes Atlas. 6th edn. 2015. https://www.idf.org/sites/default/files/EN 6E Atlas_Full_0.pdf (accessed 07 Dec 2015).

7. World Health Organisation. Sexual and reproductive health. 2015. http://www.who.int/reproductivehealth/topics/infertility/definitions/en/ (accessed 13 Nov 2015)

8. Clausen TD, Mathiesen ER, Hansen T, et al. High prevalence of type 2 diabetes and pre-diabetes in adult offspring of women with gestational diabetes mellitus or type 1 diabetes: the role of intrauterine hyperglycemia. Diabetes Care 2008;31:340-6.

9. $\mathrm{Ng} \mathrm{M}$, Fleming $\mathrm{T}$, Robinson $\mathrm{M}$, et al. Global, regional, and national prevalence of overweight and obesity in children and adults during 1980-2013: a systematic analysis for the Global Burden of Disease Study 2013. Lancet 2014;384:766-81.

10. Steyn NP, Mchiza ZJ. Obesity and the nutrition transition in sub-Saharan Africa. Ann N Y Acad Sci 2014;1311:88-101.

11. Treloar C, Porteous J, Hassan F, et al. The cross cultural context of obesity: an INCLEN multicentre collaborative study. Health Place 1999;5:279-86.

12. Walker AR, Adam F, Walker BF. World pandemic of obesity: the situation in Southern African populations. Public Health 2001;115:368-72.

13. Neeland IJ, Turer AT, Ayers CR, et al. Dysfunctional adiposity and the risk of prediabetes and type 2 diabetes in obese adults. JAMA 2012;308:1150-9.

14. Hossain P, Kawar B, El Nahas M. Obesity and diabetes in the developing world-a growing challenge. N Engl J Med 2007;356:213-15.

15. Heliovaara M, Aromaa A. Parity and obesity. J Epidemiol Commun Health 1981;35:197-9.

16. Kritz-Silverstein D, Barrett-Connor E, Wingard DL. The effect of parity on the later development of non-insulin-dependent diabetes mellitus or impaired glucose tolerance. $N$ Engl J Med 1989;321:1214-9.

17. Gunderson EP, Abrams B. Epidemiology of gestational weight gain and body weight changes after pregnancy. Epidemiol Rev 1999;21:261-75.

18. Hoque M, Hoque E, Anwar M, et al. Assessment and comparison of obstetric characteristics and perinatal outcomes of rural population of South Africa. Trop J Obstet Gynaecol 2013;26:60-7.

19. Nergard CS, Ho TPT, Diallo D, et al. Attitudes and use of medicinal plants during pregnancy among women at healthcare centers in three regions of Mali, West-Africa. J Ethnobiol Ethnomed $2015 ; 11: 1$. 
20. Harrison A, Colvin CJ, Kuo C, et al. Sustained high HIV incidence in young women in Southern Africa: social, behavioral, and structural factors and emerging intervention approaches. Curr HIVIAIDS Rep 2015:12:207-15.

21. Karamchand S, Leisegang R, Schomaker M, et al. Risk factors for incident diabetes in a cohort taking first-line nonnucleoside reverse transcriptase inhibitor-based antiretroviral therapy. Medicine (Baltimore) 2016;95:e2844.

22. Shamseer L, Moher D, Clarke M, et al. Preferred reporting items for systematic review and meta-analysis protocols (PRISMA-P) 2015: elaboration and explanation. BMJ 2015;349:g7647.

23. World Health Organization. Dept. of Noncommunicable Disease Surveillance. Definition, diagnosis and classification of diabetes mellitus and its complications. Geneva: World Health Organization, 1999.
24. Hoy $D$, Brooks $P$, Woolf $A$, et al. Assessing risk of bias in prevalence studies: modification of an existing tool and evidence of interrater agreement. J Clin Epidemiol 2012;65:934-9.

25. Borenstein $\mathrm{MH}$, Higgins LV, Rothstein JPTHR. Introduction to meta-analysis. Vol. 10. Chichester, UK: Wiley, 2009: 187-204.

26. Higgins JP, Altman DG, Gotzsche PC, et al. The Cochrane Collaboration's tool for assessing risk of bias in randomised trials. BMJ 2011;343:d5928

27. Egger M, Smith GD. Meta-analysis. Potentials and promise. BMJ 1997;315:1371-4.

28. Begg CB, Mazumdar M. Operating characteristics of a rank correlation test for publication bias. Biometrics 1994;50:1088-101.

29. Taylor S, Tweedie R. Trim and fill: a simple funnel plot based method of testing and adjusting for publication bias in meta-analyses. Fort Collins, CO: Colorado State University, 1998. 